\title{
Effects of corn steep liquor on $\beta$-poly(L-malic acid) production in Aureobasidium melanogenum
}

Genan Wang ${ }^{1,2}$, Bingyi Shi ${ }^{1,2}$, Pan Zhang ${ }^{1,2}$, Tingbin Zhao ${ }^{4}$, Haisong Yin ${ }^{1,2,3^{*}}$ and Changsheng Qiao ${ }^{1,2,4^{*}}$ (D)

\begin{abstract}
$\beta$-poly(L-malic acid) (PMLA) is a water-soluble biopolymer used in medicine, food, and other industries. However, the low level of PMLA biosynthesis in microorganisms limits its further application in the biotechnological industry. In this study, corn steep liquor (CSL), which processes high nutritional value and low-cost characteristics, was selected as a growth factor to increase the PMLA production in strain, Aureobasidium melanogenum, and its metabolomics change under the CSL addition was investigated. The results indicated that, with $3 \mathrm{~g} / \mathrm{L} C S L$, PMLA production, cell growth, and yield $\left(Y_{p / x}\right)$ were increased by $32.76 \%, 41.82 \%$, and $47.43 \%$, respectively. The intracellular metabolites of $A$. melanogenum, such as amino acids, organic acids, and key intermediates in the TCA cycle, increased after the addition of CSL, and the enrichment analysis showed that tyrosine may play a major role in the PMLA biosynthesis. The results presented in this study demonstrated that the addition of CSL would be an efficient approach to improve PMLA production.
\end{abstract}

Keywords: $\beta$-poly(L-malic acid) (PMLA), Corn steep liquor, Aureobasidium melanogenum, Metabolomics

\section{Key points}

- CSL can benefit the $\beta$-poly(L-malic acid) production.

- CSL influences the metabolism of the amino acids.

- CSL could be used as an economic nitrogen source for PMLA production.

\section{Introduction}

Polymalic acid (PMLA) is a polyester of L-malic acid with a wide range of applications in the medical, food, and environmental industries due to its excellent biochemical properties, including biocompatibility, biodegradability, and chemical modifiability (Zeng et al. 2019). Several chemical synthesis routes are available in the production

\footnotetext{
*Correspondence: 371954887@qq.com; qiaochangsheng@163.com

1 Key Laboratory of Industrial Fermentation Microbiology (Tianjin University of Science and Technology), Ministry of Education,

Tianjin 300457, People's Republic of China

Full list of author information is available at the end of the article
}

of PMLA (Kajiyama et al. 2004; Portilla-Arias et al. 2008; Vert 1998), but these methods are costly, polluted, and difficult to scale up for commercial applications. Microorganisms, such as Aureobasidium melanogenum, can also produce PMLA from sugar during fermentation at high production rates, optical purity, and high molecular weights suitable in many applications (Zou et al. 2019).

Regardless of the microorganism used in PMLA production, L-malic acid is the only precursor in PMLA biosynthesis (Zeng et al. 2019). The three major metabolic pathways in PMLA biosynthesis are the tricarboxylic acid cycle (TCA), reductive TCA (rTCA), and glyoxylate pathway (Chi et al. 2016). In recent years, several factors were tested to increase PMLA production, including the screening of mutant strains, optimizing fermentation conditions, selecting suitable carbon sources, and adding growth factors (Cao et al. 2019a, b). Moreover, the researcher speculated that the metabolic pathway of PMLA may vary in different strains. The research found that PMLA production is considerably associated with
Springer Open (c) The Author(s) 2020. This article is licensed under a Creative Commons Attribution 4.0 International License, which permits use, sharing, adaptation, distribution and reproduction in any medium or format, as long as you give appropriate credit to the original author(s) and the source, provide a link to the Creative Commons licence, and indicate if changes were made. The images or other third party material in this article are included in the article's Creative Commons licence, unless indicated otherwise in a credit line to the material. If material is not included in the article's Creative Commons licence and your intended use is not permitted by statutory regulation or exceeds the permitted use, you will need to obtain permission directly from the copyright holder. To view a copy of this licence, visit http://creativeco mmons.org/licenses/by/4.0/. 
the glyoxylate pathway in Physarum polycephalum with the addition of intermediates and inhibitors (Lee et al. 1999), and it can also be catalyzed by nonbiotin-dependent carboxylation, which is related to the rTCA pathway in A. pullulans (Cao et al. 2014). Due to the varied biosynthesis pathways for PMLA production, it is challenging to construct genetically engineered strains. As a result, selecting a suitable growth factor becomes an easy way to improve PMLA production.

Corn steep liquor (CSL) is a by-product of the corn wet-milling industry that contains nutrients essential for microbial growth. It has been proposed as a potentially effective substrate for many target metabolites produced by microorganisms (Amado et al. 2017). Researchers found that CSL increases the production of citric acid and calcium malate in Yarrowia lipolytica (Cavallo et al. 2020) and Penicillium viticola (Khan et al. 2014), respectively. However, in recent years research of PMLA biosynthesis, little attention has been paid to investigate the mechanism from a metabolomics aspect.

Gas chromatography-mass spectrometry (GC-MS) is a widely used analytical technique with high separation efficiency and sensitivity detection in resolving complex biological mixtures (He et al. 2018). Moreover, the intracellular metabolites produced by microorganisms influenced by different growth factors could be detected via GC-MS. The researcher investigated the various effects of deregulating enzymes on the metabolites between engineered L-lysine-producing Corynebacterium glutamicum and the wild-type strain through intracellular metabolite profiles (da Luz et al. 2017). Some other researchers combined intracellular metabolites with proteomics to identify the difference between marine sediment (Beale et al. 2017) and trace pollution (Beale et al. 2018). This approach could easily detect the main difference between microorganisms under different conditions or growth factors.

In this study, we aimed to explore the effect of CSL on the enhancement of cell growth and PMLA production in A. melanogenum. Metabolomics technology was used to gain insight into the working mechanism and to analyze the change in the key intracellular metabolites of $A$. melanogenum after CSL addition. The results will help to determine an efficient approach to improve PMLA production.

\section{Materials and methods}

\section{Microorganism and medium}

Aureobasium melanogenum CGMCC18996 was isolated in our laboratory and then preserved in the China General Microbiological Culture Collection Center (Beijing, China No. CGMCC18996). The strain was stored in potato dextrose agar (PDA) slants at $4{ }^{\circ} \mathrm{C}$ and subcultured every 2 weeks. The seed medium contained $60 \mathrm{~g} / \mathrm{L}$ sucrose, $3 \mathrm{~g} / \mathrm{L}$ yeast extract, $2 \mathrm{~g} / \mathrm{L}$ succinic acid, $1 \mathrm{~g} / \mathrm{L}$ ammonium sulfate, $0.4 \mathrm{~g} / \mathrm{L} \mathrm{K}_{2} \mathrm{CO}_{3}, 0.1 \mathrm{~g} / \mathrm{L} \mathrm{KH}_{2} \mathrm{PO}_{4}$, $0.1 \mathrm{~g} / \mathrm{L} \mathrm{MgSO}_{4}, 0.05 \mathrm{~g} / \mathrm{L} \mathrm{ZnSO}_{4}$, and $0.1 \% \mathrm{CSL}(\mathrm{V} / \mathrm{V})$. The fermentation medium contained $180 \mathrm{~g} / \mathrm{L}$ sucrose, $35 \mathrm{~g} / \mathrm{L}$ peptone, $0.1 \mathrm{~g} / \mathrm{L} \mathrm{KH}_{2} \mathrm{PO}_{4}, 2 \mathrm{~g} / \mathrm{L} \mathrm{NaNO}, 0.3 \mathrm{~g} / \mathrm{L}$ $\mathrm{MgSO}_{4}, 0.5 \mathrm{~g} / \mathrm{L} \mathrm{KCl}, 0.05 \mathrm{~g} / \mathrm{L} \mathrm{MnSO}_{4}$, and $20 \mathrm{~g} / \mathrm{L} \mathrm{CaCO}_{3}$. Both seed and fermentation mediums were sterilized at $121{ }^{\circ} \mathrm{C}$ for $20 \mathrm{~min}$ before use.

The amino acids and vitamins in CSL were determined via the methods of Culea et al. (2015) and Klejdus et al. (2004).

\section{Fermentation conditions}

The primary seed culture of Aureobasidium. melanogenum CGMCC18996 was prepared by inoculating cells grown on solid medium into $500 \mathrm{~mL}$ Erlenmeyer flasks containing $100 \mathrm{~mL}$ seed culture medium and then cultured at $25^{\circ} \mathrm{C}$ for approximately $40 \mathrm{~h}$ in a rotary shaker (IS-RDS3, Crystal Technology and Industries, Inc., USA). CSL at 1, 3, 5, 7, and $9 \mathrm{~g} / \mathrm{L}$ was placed into $500 \mathrm{~mL}$ Erlenmeyer flasks containing $100 \mathrm{~mL}$ fermentation medium with primary seed culture $(10 \%, \mathrm{v} / \mathrm{v})$, and fermentation cultivation was conducted at $25{ }^{\circ} \mathrm{C}$ for $144 \mathrm{~h}$ in a rotary shaker at $200 \mathrm{rpm}$. Fed-batch fermentation kinetics was investigated in a $5 \mathrm{~L}$ stirred tank fermenter (GRJB-5D, Zhenjiang Gree Co., Ltd., China) containing 3 L fermentation medium inoculated with $300 \mathrm{~mL}$ seed culture, and the fermentation medium was operated at $25^{\circ} \mathrm{C}$ for $156 \mathrm{~h}$ with an agitation speed and aeration rate of $500 \mathrm{rpm}$ and $1.3 \mathrm{vvm}$, respectively.

\section{Assay of PMLA production}

Fermentation broth $(10 \mathrm{~mL})$ was collected at different time points and centrifuged at $10,000 \times g$. The resulting supernatant $(5 \mathrm{~mL})$ was mixed with $5 \mathrm{~mL} 2 \mathrm{M} \mathrm{H}_{2} \mathrm{SO}_{4}$ and then incubated at $110{ }^{\circ} \mathrm{C}$ for $11 \mathrm{~h}$. After neutralization, the sample was analyzed with HPLC (L-2000, Hitachi Ltd., Japan) by using a Prevail $\mathrm{C} 18$ organic acid column at $25{ }^{\circ} \mathrm{C}$ eluted with $25 \mathrm{mM} \mathrm{KH_{2 }} \mathrm{PO}_{4}$ at a rate of $1.0 \mathrm{~mL} /$ min. The PMLA concentration was determined by comparing the difference in L-malic acid concentrations before and after hydrolysis.

\section{Assay of fermentation parameters}

Cell density was determined via the method of dry cell weight (DCW) in three steps. Prior to measurement, $\mathrm{HCl}(3 \mathrm{M})$ was added to $10 \mathrm{~mL}$ of fermentation broth to eliminate the excess concentration of $\mathrm{CaCO}_{3}$. The fermentation broth $(10 \mathrm{~mL})$ was centrifuged at $8000 \times g$ for $10 \mathrm{~min}$, and the resulting precipitate was washed twice with phosphate buffer saline (PBS) buffer. After 
recentrifugation, the precipitates were dried overnight at $80{ }^{\circ} \mathrm{C}$ and then weighed.

The residual sugar was analyzed with the 3,5-dinitrosalicylic acid assay (Miller 1959).

\section{Specific growth rate, PMLA productivity, and PMLA yield $\left(\mathrm{Y}_{\mathrm{p} / \mathrm{x}}\right)$ calculation}

The specific growth rate was measured using the increased biomass versus interval time (Liu et al. 2005), and $\mathrm{Y}_{p / x}$ was measured by determining the ratio of increased PMLA to the increased cell biomass concentration over the interval time (Yin et al. 2019).

\section{Extraction of intracellular metabolites and metabolomics analysis}

In the extraction of intracellular metabolites, three independent fermentation broth samples $(50 \mathrm{~mL})$ at the fermentation times of $72,96,120$, and $144 \mathrm{~h}$ were collected from the $5 \mathrm{~L}$ stirred tank fermenter. The excess concentration of $\mathrm{CaCO}_{3}$ that was not consumed by $A$. melanogenum was removed by centrifuging the samples at $8000 \times g$ for $30 \mathrm{~s}$. The resulting supernatant was centrifuged at $10,000 \times g$ for $5 \mathrm{~min}$. The precipitate was collected, washed twice with normal saline at $-4{ }^{\circ} \mathrm{C}$, and ground in liquid nitrogen for $25 \mathrm{~min}$. The cell fragment $(200 \mathrm{mg}$ ) of liquid nitrogen grind was collected and mixed with $1 \mathrm{~mL}$ of precooling methanol (60\%). The mixture was then centrifuged at $8000 \times g$ for $5 \mathrm{~min}$. After the derivatization process, it was subjected to refrigerated centrifugation at $8000 \times g$ for $5 \mathrm{~min}$, and the resulting supernatant was prepared for GC-MS analysis after storage for $2 \mathrm{~h}$ at $25^{\circ} \mathrm{C}$.

\section{Data processing and analysis}

GC-MS files (.MS) were converted to AIA (.CDF) format for XCMS online (Gowda et al. 2014) analysis. Peak detection and alignment were measured by default centwave method for GC Single Quadruple (Agilent 7980A/5975C, GC-MSD), and the METLIN database was used for Metabolites identification.

GC-MS pre-processing data were written as .csv files and imported to the MetaboAnalyst (Chong et al. 2019) for data normalization. The peak intensity represented the relative concentration, and the downstream analysis was performed by $\mathrm{R}$ studio using package BiocManager version 1.30.10.

\section{Results}

\section{Effect of CSL addition on PMLA production of $A$.} melanogenum

In this study, the effects of different CSL concentrations on PMLA production of $A$. melanogenum that cultured in a rotary shaker were evaluated. The accumulation of biomass increased with the increasing CSL concentration, and the highest PMLA production, $69.8 \mathrm{~g} / \mathrm{L}$, was obtained with the addition of $3 \mathrm{~g} / \mathrm{L}$ CSL, revealing a $36.8 \%$ increase compared to the control (Fig. 1). The effect of CSL on the PMLA production of A. melanogenum was further verified by culturing $A$. melanogenum in a $5 \mathrm{~L}$ fermenter (Fig. 2), and the results indicated that the addition of $3 \mathrm{~g} / \mathrm{L}$ CSL facilitated the growth and PMLA production of $A$. melanogenum. After $156 \mathrm{~h}$ of fermentation, PMLA production reached $73.72 \mathrm{~g} / \mathrm{L}$, as shown in Fig. 2a, which was $32.76 \%$ higher than that of the control group. The biomass reached $62.83 \mathrm{~g} / \mathrm{L}$, as shown in Fig. 2b, which was $41.82 \%$ higher than that of the control group. Meanwhile, after $24 \mathrm{~h}$ of fermentation, the highest specific growth rate reached $0.19 \mathrm{~h}^{-1}$, as shown in Fig. 2c, which was $37.72 \%$ higher than that of the control group. However, a rapid decline in the specific growth rate was detected after $24 \mathrm{~h}$ when no distinct difference existed between the control and CSL groups. The PMLA yield $\left(\mathrm{Y}_{p / x}\right)$ in CSL showed a significant increase in the late stage of fermentation, as shown in Fig. 2d. After $120 \mathrm{~h}$, the highest $Y_{p / x}$ reached $1.82 \mathrm{~g} / \mathrm{g}$, which was $47.43 \%$ higher than that of the control group. Moreover, the residual sugar (Fig. 2e) was consumed rapidly after the addition of $3 \mathrm{~g} / \mathrm{L} \mathrm{CSL}$, and the end time was $12 \mathrm{~h}$ earlier than that of the control.

\section{Metabolomics analysis of $A$. melanogenum cultivated with the addition of $3 \mathrm{~g} / \mathrm{L} \mathrm{CSL}$}

The PLS-DA scores plot (Fig. 3) showed a clear variation in the metabolite profiles under both groups, and the metabolomics data revealed a total of 36 metabolites of $A$. melanogenum that were detected via GC-MS at $72,96,120$, and $144 \mathrm{~h}$ time points (Fig. 4). Among them, most of the metabolite concentrations in the CSL group

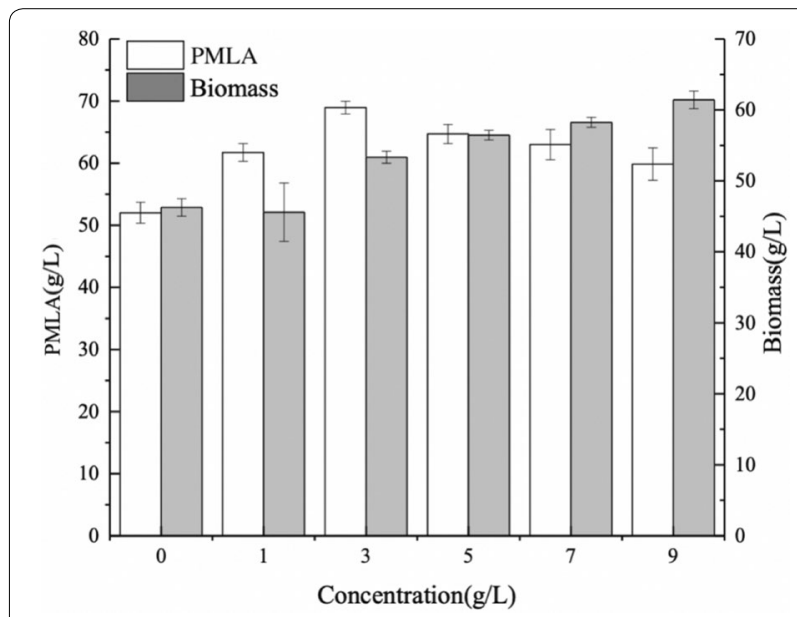

Fig. 1 Different concentrations of CSL on PMLA production and biomass 

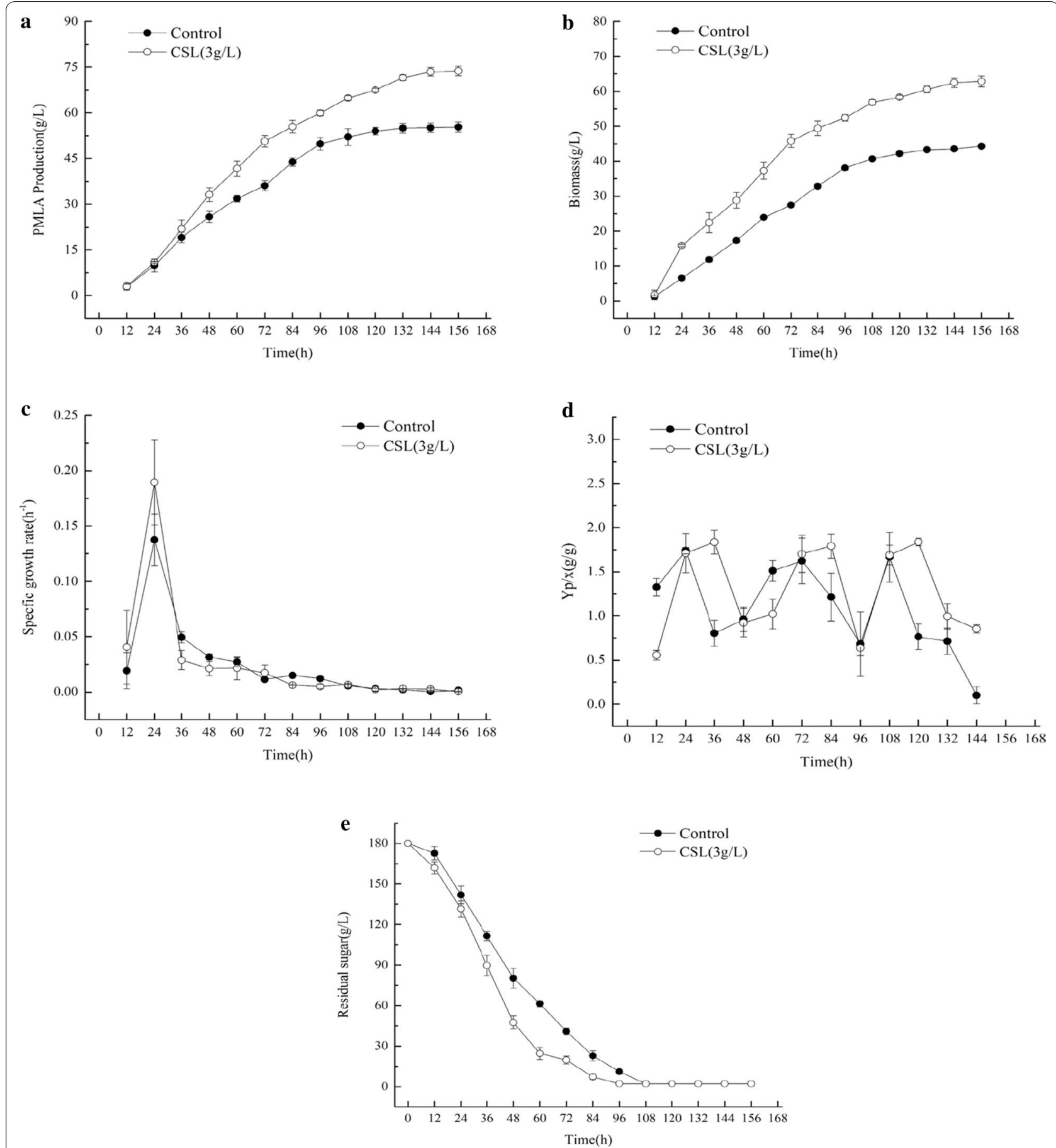

Fig. 2 PMLA production versus a biomass, $\mathbf{b}$ specific productivity rate, $\mathbf{c}$ specific growth rate, $\mathbf{d}$ PMLA yield, and e residual sugar in the $5 \mathrm{~L}$ fermenter

were increased compared to the control, especially at $120 \mathrm{~h}$ (Fig. 4). The enrichment analysis (Additional file 1: Figure S1) indicated that the concentrations of metabolite related to PMLA biosynthesis increased obviously. These metabolites were deoxyinosine, homogentisate, fumarate acid, and 5-aminolevulinic, which involved in the purine metabolism $(\mathrm{P}<0.05)$, tyrosine metabolism $(\mathrm{P}<0.05)$, TCA cycle $(\mathrm{P}<0.05)$, and glycine and serine metabolism. 


\section{Scores Plot}

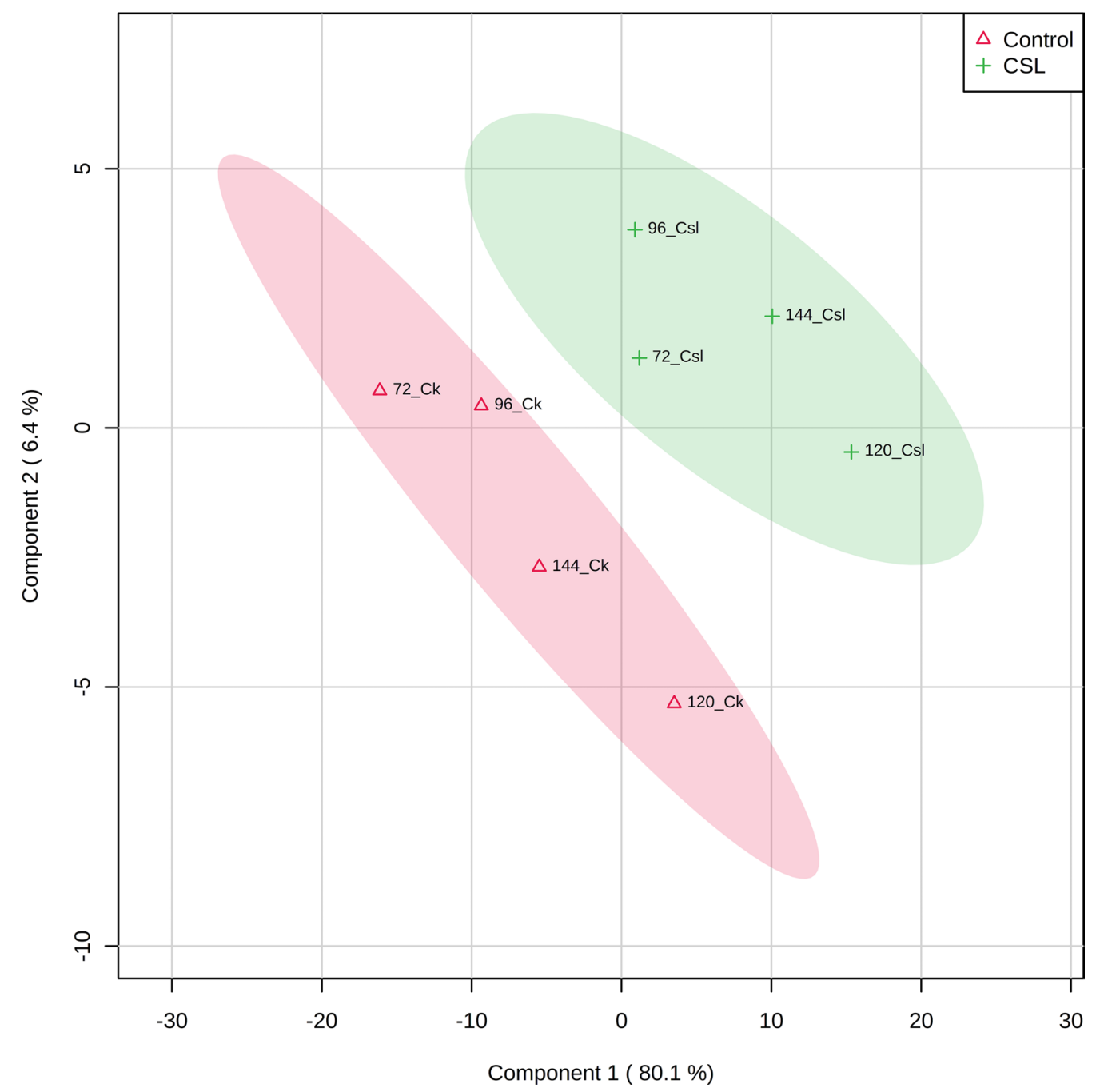

Fig. 3 PLS-DA score plot of the control and CSL

\section{Metabolomics analysis of PMLA metabolic pathway}

The metabolic pathway related to PMLA biosynthesis and the relative concentration changes were depicted (Fig. 5). Compared to the control, nearly all of the metabolite concentrations increased and peaked at $120 \mathrm{~h}$. The concentrations of six metabolites related to the tryptophan metabolism, which were serotonin, 5-methoxytryptamine, indole-3-acetonitrile, 2-aminomuconate semialde hyde, L-kynurenine, and kynurenate, were improved. The addition of CSL caused the accumulation of 5-aminolevulinate that involved in the glycine and serine metabolism. Moreover, the concentrations of homogentisate and R-reticuline, which related to the tyrosine metabolism, and D-lactaldehyde that would enter the TCA cycle were increased. For the TCA cycle, the concentrations of fumarate and oxalosuccinate, which are the precursors of malic acid, showed an increasing effect. And the results also indicated that the concentrations of L-valine and hydroxypruvate were decreased.

\section{Assay of the amino acids on PMLA production}

The nutritional substances of CSL were tested. Seventeen kinds of amino acids and three types of vitamins were detected. Table 1 showed the ratio of each amino acid quality to the CSL quality. Among them, four amino acids (tyrosine, serine, glycine, and tryptophan) were selected based on the PMLA biosynthesis pathway (Fig. 5) and added to the fermentation broth (without peptone) separately for evaluating the influence on PMLA production. The result revealed that the PMLA production increased 


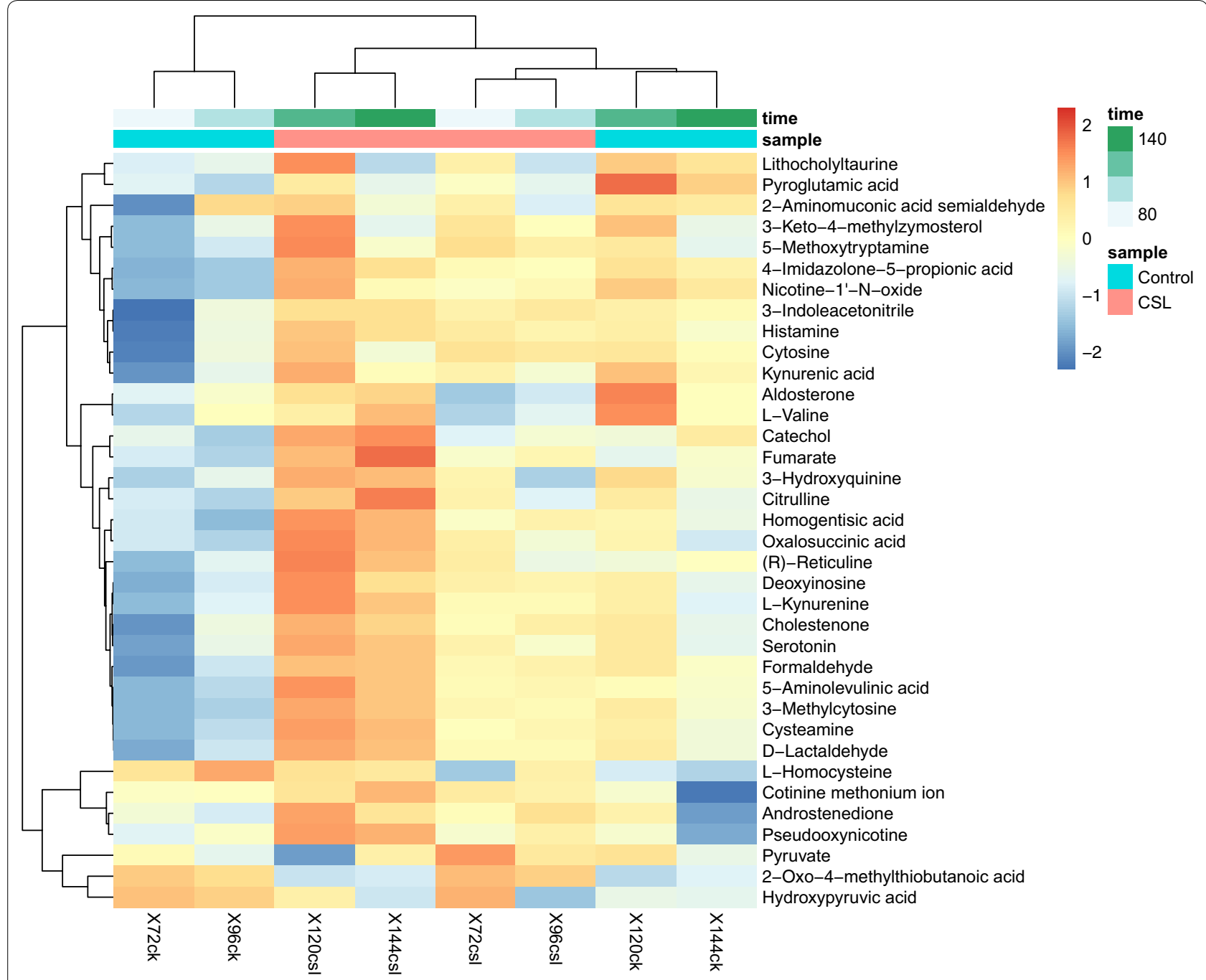

Fig. 4 Heat map of the relative concentrations of metabolites in the control and CSL

by $29.5 \%$ after adding tyrosine, $21.9 \%$ after adding serine, 9.3\% after adding tryptophan, 7.6\% after adding glycine, and $39.47 \%$ after adding CSL (Fig. 6). Moreover, significant changes were observed between the tyrosine group and the control $(\mathrm{P}<0.01)$, and the CSL and the control $(\mathrm{P}<0.01)$.

\section{Discussion}

Using the growth factor is an economical way to improve PMLA production (Cao et al. 2019b). In this study, the addition of $3 \mathrm{~g} / \mathrm{L}$ CSL facilitates cell growth and PMLA production in A. melanogenum. The maximal PMLA production and biomass increased by $32.76 \%$ and $41.82 \%$, respectively. Furthermore, the specific growth rate revealed a rapid increase during the early phase of fermentation, and $\mathrm{Y}_{p / x}$ in the CSL group was higher than that of the control in the late stage.
Moreover, CSL contained various amino acids and vitamins (Table 1) that could function as an effective stimulatory agent of cell growth and PMLA biosynthesis. It was found that the rich nutrients of CSL became a source of nitrogen, essential minerals, and cofactors required for Pichia pastoris cell growth (Zheng et al. 2012). Moreover, the addition of CSL increased the biomass of Trametes versicolor in the initial $12 \mathrm{~h}$ of fermentation, and an accelerated growth rate was observed (Wang et al. 2014). The addition of $3 \mathrm{~g} / \mathrm{L}$ CSL provided various amino acids and vitamins (Table 1 ), indicating that the CSL could be an effective stimulatory agent of cell growth in A. melanogenum. The high cell growth of $A$. melanogenum led to a high level of PMLA production. Therefore, we speculated that various nutritional substances provided by CSL benefit cell growth, which in turn benefit PMLA production. 


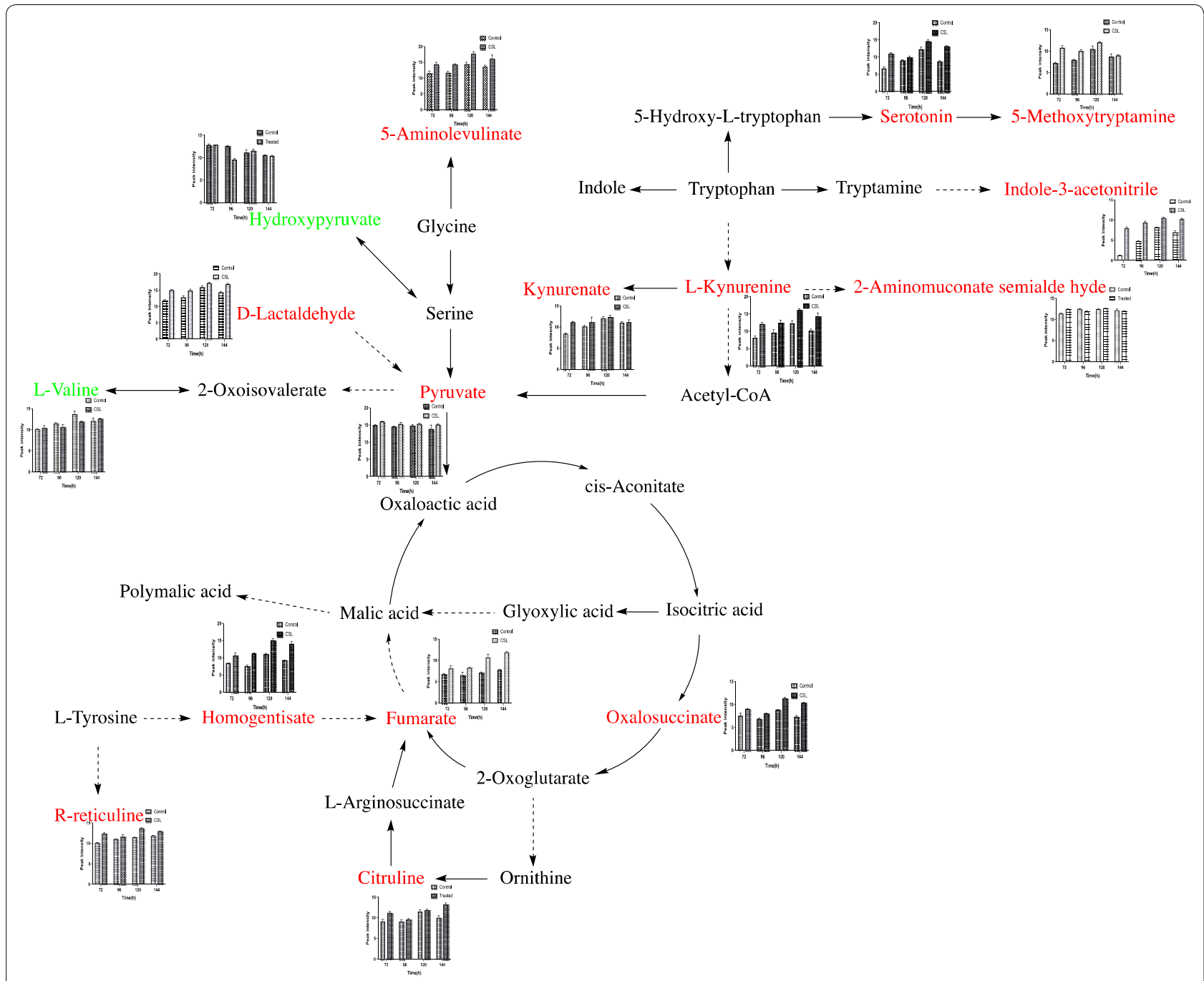

Fig. 5 Metabolic pathway related to the PMLA biosynthesis based on the metabolomics data (color in red and green represent up-regulated and down-regulated metabolites)

Table 1 Nutritional substances contained in the corn steep liquor

\begin{tabular}{|c|c|c|c|c|c|}
\hline Name & Unit & Test result & Name & Unit & Test result \\
\hline \multicolumn{6}{|l|}{ Amino acids } \\
\hline Aspartic acid & $\%$ & 2.22 & Alanine & $\%$ & 3.84 \\
\hline Threonine & $\%$ & 1.20 & Valine & $\%$ & 1.98 \\
\hline Serine & $\%$ & 1.28 & Methionine & $\%$ & 0.72 \\
\hline Glutamate & $\%$ & 5.41 & Isoleucine & $\%$ & 1.21 \\
\hline Glycine & $\%$ & 2.05 & Leucine & $\%$ & 3.40 \\
\hline Tyrosine & $\%$ & 0.62 & Phenylalanine & $\%$ & 1.38 \\
\hline Lysine & $\%$ & 1.16 & Histidine & $\%$ & 1.16 \\
\hline Arginine & $\%$ & 1.51 & Proline & $\%$ & 3.20 \\
\hline Tryptophan & $\%$ & 0.17 & & & \\
\hline \multicolumn{6}{|l|}{ Vitamins } \\
\hline Vitamin $B_{2}$ & mg/kg & 11.40 & Choline & $\mathrm{mg} / 100 \mathrm{~g}$ & 344 \\
\hline Inositol & $\mathrm{mg} / 100 \mathrm{~g}$ & 226 & & & \\
\hline
\end{tabular}




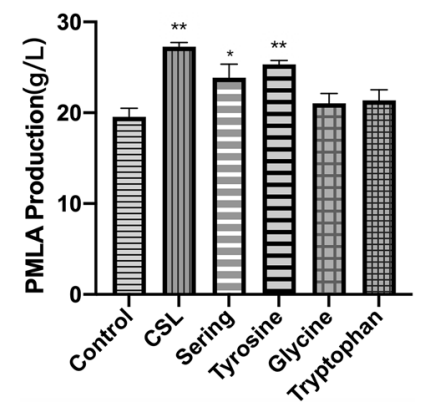

Fig. 6 Different amino acids on PMLA production $\left({ }^{*} P<0.05,{ }^{* *} P<0.01\right.$ versus the control group by the LSD analysis)

With the help of metabolomics technology, the working mechanism underlying the effect of the addition of $3 \mathrm{~g} / \mathrm{L}$ CSL on the metabolism of $A$. melanogenum was analyzed. The metabolomics data showed that the relative concentrations of metabolites involved in amino acid and organic acid metabolism were changed during fermentation (Fig. 5). The PLS-DA showed a clear separation between the two groups. Moreover, the tyrosine metabolism, glycine and serine metabolism, and TCA cycle were enhanced after the $3 \mathrm{~g} / \mathrm{L}$ CSL addition. Among them, for the tyrosine metabolism, tyrosine first converted to 4-hydroxyphenylpyruvate then to homogentisate, which further converted to fumarylacetoacetate, and fumarylacetoacetate would transfer to fumarate that entered the TCA cycle (Wang et al. 2019). The metabolomics data showed that the relative concentration of homogentisate increased by 1.44 -fold, and fumarate increased by $1.52-$ fold at $144 \mathrm{~h}$ compared to the control. The concentration of 5-aminolevulinate, related to the glycine and serine metabolism, increased about 1.2-fold. And 2-aminomuconate semialde hyde concentration increased about 1.3-fold, which can lead tryptophan into the glutaric acid pathway and then the TCA cycle (Fukuwatari et al. 2001). The result also proved that deoxyinosine, involved in purine metabolism, increased by 1.2 -fold, and this metabolism is related to the cell's nitrogen absorption that can provide molecules essential for DNA and RNA biosynthesis, energy metabolism, and signal transduction (Jessica and James 2017). The 5L-fermenter data showed that the maximal PMLA production was obtained after 120 h (Fig. 2a), but yield (Fig. 2e) was decreased after $120 \mathrm{~h}$. These results were consistent with the metabolomics data that the metabolites reached the highest at $120 \mathrm{~h}$ then it began to decrease.

Metabolomics data can express the metabolic changes under different conditions. However, after the peak alignment and the data normalization, some of the target-metabolites may lose in the data. Therefore, we speculated that the concentrations of tyrosine, glycine, serine, and tryptophan, which related to the tyrosine pathway, glycine and serine pathway, and tryptophan pathway, were improved in the CSL group. These pathways may further increase the PMLA production. Consequently, we tested the nutritional substances of CSL and found 17 kinds of amino acids and three types of vitamins (Table 1). Among them, serine is $1.28 \%$, tyrosine is $0.62 \%$, tryptophan is $0.17 \%$, and glycine is $2.05 \%$ of the total CSL weight.

In order to figure out which amino acid most benefiting PMLA production in the CSL, different amino acids related to the up-regulated pathway (Fig. 5) were added to the fermentation broth based on the ratio of their quality to the total CSL quality to evaluate the effect on PMLA production. The result demonstrated that these four amino acids increased PMLA production by 29.5\% (tyrosine), 21.9\% (serine), 9.3\% (tryptophan), and 7.6\% (glycine), respectively. Apart from that, a significant change between the tyrosine group and the control $(\mathrm{P}<0.01)$ was observed, and this result is consistent with the enrichment analysis (Additional file 1: Figure S1), indicating that the CSL significantly influenced the tyrosine metabolism $(\mathrm{P}<0.05)$. The data obtained from the PMLA-related pathway (Fig. 5) suggested that tyrosine flows to the TCA cycle by converted to fumarate, which is further converted to malic acid that increases the production of PMLA. Meanwhile, it also indicated that all of the four amino acids, tyrosine, glycine, serine, and tryptophan, eventually flows to the TCA cycle (Fig. 5). However, considering the relative concentration changes of the metabolites (pyruvate and fumarate), we can speculate that tyrosine may play a crucial role in increasing PMLA production under the CSL.

As mentioned above, the results showed that the TCA cycle was up-regulated after the addition of $3 \mathrm{~g} / \mathrm{L} \mathrm{CSL}$, which would further increase PMLA production. Therefore, we can conclude that the up-regulated TCA cycle is the key metabolic pathway under the $3 \mathrm{~g} / \mathrm{L} \mathrm{CSL}$ for the increase of PMLA production among three speculated metabolic pathways, i.e., the glyoxylate acid pathway, the reductive TCA pathway, and the TCA pathway (Zou et al. 2019). Meanwhile, the energy provided by the up-regulated purine metabolism may accelerate this process.

The improvement in amino acid and organic acid metabolism in A. melanogenum from the addition of $3 \mathrm{~g} / \mathrm{L}$ CSL generated various amino acids and organic acids to improve cell growth. The conversions of the metabolites related to the TCA cycle were enhanced after the addition of $3 \mathrm{~g} / \mathrm{L}$ CSL. Therefore, we speculated that CSL is an effective stimulatory agent for cell growth and PMLA biosynthesis in A. melanogenum. Meanwhile, CSL could be used as an economic nitrogen source due to its high nutrition and low cost. As a potential 
replacement of peptone and yeast extract in PMLA production, CSL has satisfactory use prospects in the PMLA industry.

\section{Supplementary information}

Supplementary information accompanies this paper at https://doi. org/10.1186/s13568-020-01147-8.

Additional file 1: Figure S1. Enrichment analysis of metabolomics data.

\section{Acknowledgements}

Not applicable.

\section{Authors' contributions}

$\mathrm{CQ}$ and $\mathrm{HY}$ conceived and designed research, GW, BS, and PZ conducted experiments. TZ contributed new reagents or analytical tools. GW analyzed data and wrote the manuscript. All authors read and approved the final manuscript.

\section{Funding}

This study was funded by the Tianjin Municipal Science and Technology Commission (No. 17PTGCCX00190, No. 17PTSYJC00080, No. 17YFCZZC00310 and No. 16YFXTSF00460) and the Tianjin Engineering Research Center of Microbial Metabolism and Fermentation Process Control (No. ZXKF20180301).

\section{Availability of data and materials}

All data generated or analyzed during this study are included in this paper.

\section{Ethics approval and consent to participate}

Not applicable.

\section{Consent for publication}

Not applicable.

\section{Competing interests}

The authors declare that they have no competing interests.

\begin{abstract}
Author details
${ }^{1}$ Key Laboratory of Industrial Fermentation Microbiology (Tianjin University of Science and Technology), Ministry of Education, Tianjin 300457, People's Republic of China. ${ }^{2}$ Tianjin Engineering Research Center of Microbial Metabolism and Fermentation Process Control, College of Biotechnology, Tianjin University of Science and Technology, Tianjin 300457, People's Republic of China. ${ }^{3}$ School of Bioengineering, Tianjin Modern Vocational Technology College, Tianjin 300350, People's Republic of China. ${ }^{4}$ Tianjin Huizhi Biotrans Bioengineering Co., Ltd., Tianjin 300457, People's Republic of China.
\end{abstract}

Received: 7 July 2020 Accepted: 15 November 2020

Published online: 01 December 2020

\section{References}

Amado IR, Vázquez JA, Pastrana L, Teixeira JA (2017) Microbial production of hyaluronic acid from agro-industrial by-products: molasses and corn steep liquor. Biochem Eng J 117:181-187. https://doi.org/10.1016/j. bej.2016.09.017

Beale DJ, Crosswell J, Karpe AV, Ahmed W, Williams M, Morrison PD, Metcalfe S, Staley C, Sadowsky MJ, Palombo EA, Steven ADL (2017) A multi-omics based ecological analysis of coastal marine sediments from Gladstone, in Australia's Central Queensland, and Heron Island, a nearby fringing platform reef. Sci Total Environ 609:842-853. https://doi.org/10.1016/j. scitotenv.2017.07.184

Beale D, Crosswell J, Karpe A, Metcalfe SS, Morrison P, Staley C, Ahmed W, Sadowsky M, Palombo E, Steven A (2018) Seasonal metabolic analysis of marine sediments collected from Moreton Bay in South East Queensland,
Australia, using a multi-omics-based approach. Sci Total Environ 631:1328-1341. https://doi.org/10.1016/j.scitotenv.2018.03.106

Cao W, Luo J, Qi B, Zhao J, Qiao C, Ding L, Su Y, Wan Y (2014) $\beta$-poly(l-malic acid) production by fed-batch culture of Aureobasidium pullulans ipe-1 with mixed sugars. Eng Life Sci 14(2):180-189. https://doi.org/10.1002/ elsc.201200189

Cao W, Cao W, Shen F, Luo J, Yin J, Qiao C, Yinhua W (2019a) Membraneassisted $\beta$-poly( $(\mathrm{L}-\mathrm{malic}$ acid) production from bagasse hydrolysates by Aureobasidium pullulans ipe-1. Bioresour Technol 295:122260. https://doi. org/10.1016/j.biortech.2019.122260

Cao W, Wang Y, Shen F, Luo J, Yin J, Qiao C, Wan Y (2019b) Efficient $\beta$-poly(lmalic acid) production from Jerusalem artichoke by Aureobasidium pullulans ipe-1 immobilized in luffa sponge matrices. Bioresour Technol 288:121497. https://doi.org/10.1016/j.biortech.2019.121497

Cavallo E, Nobile M, Cerrutti P, Foresti ML (2020) Exploring the production of citric acid with Yarrowia lipolytica using corn wet milling products as alternative low-cost fermentation media. Biochem Eng J 155:107463. https://doi.org/10.1016/j.bej.2019.107463

Chi Z, Liu G-L, Liu C-G, Chi Z-M (2016) Poly(beta-L-malic acid) (PMLA) from Aureobasidium spp. and its current proceedings. Appl Microbiol Biotechnol 100(9):3841-3851. https://doi.org/10.1007/s00253-016-7404-0

Chong J, Wishart DS, Xia J (2019) Using MetaboAnalyst 4.0 for Comprehensive and integrative metabolomics data analysis. Curr Protoc Bioinf 68(1):e86. https://doi.org/10.1002/cpbi.86

Culea M, Scrob S, Suvar S, Podea P, Haş I, Muste S (2015) Determination of amino acids in corn seed by gas chromatography-mass spectrometry. Anal Lett. https://doi.org/10.1080/00032719.2014.930869

da Luz JA, Hans E, Frank D, Zeng A-P (2017) Analysis of intracellular metabolites of Corynebacterium glutamicum at high cell density with automated sampling and filtration and assessment of engineered enzymes for effective I-Iysine production. Eng Life Sci 17(5):512-522. https://doi.org/10.1002/ elsc.201600163

Fukuwatari T, Morikawa Y, Hayakawa F, Sugimoto E, Shibata K (2001) Influence of adenine-induced renal failure on tryptophan-niacin metabolism in rats. Biosci Biotechnol Biochem 65(10):2154-2161. https://doi.org/10.1271/ bbb.65.2154

Gowda H, Ivanisevic J, Johnson CH, Kurczy ME, Benton HP, Rinehart D, Nguyen T, Ray J, Kuehl J, Arevalo B, Westenskow PD, Wang J, Arkin AP, Deutschbauer AM, Patti GJ, Siuzdak G (2014) Interactive XCMS online: simplifying advanced metabolomic data processing and subsequent statistical analyses. Anal Chem 86(14):6931-6939. https://doi.org/10.1021/ac500734c

He Y, Zhang Z, Ma P, Ji H, Lu H (2018) GC-MS profiling of leukemia cells: an optimized extraction protocol for intracellular metabolome. Anal Methods. https://doi.org/10.1039/C7AY02578E

Jessica C, James F (2017) Purine acquisition and synthesis by human fungal pathogens. Microorganisms 5(2):33. https://doi.org/10.3390/microorgan isms5020033

Kajiyama T, Kobayashi H, Taguchi T, Saito H, Kamatsu Y, Kataoka K, Tanaka J (2004) Synthesis of activated poly (a, $\beta$-malic acid) using $N$-hydroxysuccinimide and its gelation with collagen as biomaterials. Mater Sci Eng $C$ 24:815-819. https://doi.org/10.1016/j.msec.2004.08.023

Khan I, Nazir K, Wang Z-P, Liu G-L, Chi Z-M (2014) Calcium malate overproduction by Penicillium viticola 152 using the medium containing corn steep liquor. Appl Microbiol Biotechnol 98(4):1539-1546. https://doi. org/10.1007/s00253-013-5326-7

Klejdus B, Petrlova J, Potesil D, Adam V, Mikelová R, Vacek J, Kizek R, Kubáň V (2004) Simultaneous determination of water- and fat-soluble vitamins in pharmaceutical preparations by high-performance liquid chromatography coupled with diode array detection. Anal Chim Acta 520:57-67. https ://doi.org/10.1016/j.aca.2004.02.027

Lee BS, Maurer T, Kalbitzer HR, Holler E (1999) Beta-Poly(L-malate) production by Physarum polycephalum-C-13 Nuclear magnetic resonance studies. Appl Microbiol Biotechnol 52(3):415-420. https://doi.org/10.1007/s0025 30051540

Liu Y, Liu Q-S, Tay J-H (2005) Initial conditions-dependent growth kinetics in microbial batch culture. Process Biochem 40(1):155-160. https://doi. org/10.1016/j.procbio.2003.11.052

Miller GAIL (1959) Use of dinitrosalicylic acid reagent for detection of reducing sugars. Anal Chem. https://doi.org/10.1021/ac60147a030

Portilla-Arias J, García-Alvarez M, Martinez de llarduya A, Holler E, Muñoz S, Muñoz-Guerra S (2008) Synthesis, degradability, and drug releasing 
properties of methyl esters of fungal poly( $\beta$, L-malic acid). Macromol Biosci 8:540-550. https://doi.org/10.1002/mabi.200700248

Vert M (1998) Chemical routes to poly( $\beta$-malic acid) and potential applications of this water-soluble bioresorbable poly( $\beta$-hydroxy alkanoate). Polym Degrad Stabil 59(1):169-175. https://doi.org/10.1016/S0141 -3910(97)00158-4

Wang F, Hu J-H, Guo C, Liu C-Z (2014) Enhanced laccase production by Trametes versicolor using corn steep liquor as both nitrogen source and inducer. Bioresour Technol 166:602-605. https://doi.org/10.1016/j.biort ech.2014.05.068

Wang M, Toda K, Block A, Maeda H (2019) TAT1 and TAT2 tyrosine aminotransferases have both distinct and shared functions in tyrosine metabolism and degradation in Arabidopsis thaliana. J Biol Chem 294:jbc. RA118.006539. https://doi.org/10.1074/jbc.RA118.006539

Yin H, Gao C, Ye K, Zhao T, Sun A, Qiao C (2019) Evaluation of surfactant effect on $\beta$-poly(L-malic acid) production by Aureobasidium pullulans. Biotechnol Biotechnol Equip 33(1):954-966. https://doi.org/10.1080/13102 818.2019 .1631718
Zeng W, Zhang B, Liu Q, Chen G, Liang Z (2019) Analysis of the L-malate biosynthesis pathway involved in poly(-L-malic acid) production in Aureobasidium melanogenum GXZ-6 by addition of metabolic intermediates and inhibitors. J Microbiol 57(4):281-287. https://doi.org/10.1007/ s12275-019-8424-0

Zheng J, Zhao W, Guo N, Lin F, Tian J, Wu L, Zhou H (2012) Development of an industrial medium and a novel fed-batch strategy for high-level expression of recombinant $\beta$-mananase by Pichia pastoris. Bioresour Technol 118:257-264. https://doi.org/10.1016/j.biortech.2012.05.065

Zou X, Cheng C, Feng J, Song X, Lin M, Yang S-T (2019) Biosynthesis of polymalic acid in fermentation: advances and prospects for industrial application. Crit Rev Biotechnol 39(3):408-421. https://doi.org/10.1080/07388 551.2019 .1571008

\section{Publisher's Note}

Springer Nature remains neutral with regard to jurisdictional claims in published maps and institutional affiliations.

\section{Submit your manuscript to a SpringerOpen ${ }^{\odot}$ journal and benefit from:}

- Convenient online submission

- Rigorous peer review

- Open access: articles freely available online

- High visibility within the field

- Retaining the copyright to your article

Submit your next manuscript at $\boldsymbol{\nabla}$ springeropen.com 